\title{
Les tatouages décoratifs après mastectomie pour cancer du pÿsein : une stratégie d adaptation en progression
}

Kluger, $\mathrm{N}$.

2016-12

Kluger , N 2016 , ' Les tatouages décoratifs après mastectomie pour cancer du sein : une pÿstratégie d adaptation en progression ', Annales de chirurgie plastique esthetique , vol. 61 , no. 6 , pp. 868-871 . https://doi.org/10.1016/j.anplas.2016.09.006

http://hdl.handle.net/10138/230304

https://doi.org/10.1016/j.anplas.2016.09.006

publishedVersion

Downloaded from Helda, University of Helsinki institutional repository.

This is an electronic reprint of the original article.

This reprint may differ from the original in pagination and typographic detail.

Please cite the original version. 


\title{
Les tatouages décoratifs après mastectomie pour cancer du sein : une stratégie d'adaptation en progression ${ }^{2 / 2}$
}

\author{
Decorative tattooing after mastectomy for breast cancer: \\ An uprising coping strategy
}

Les tatouages font maintenant partie intégrante du paysage actuel sociétal. Le tatouage s'affiche dans la rue, sur la plage, dans les publicités ou dans les stades. D'après un sondage datant de 2010, un français sur 10 est tatoué [1]. Les raisons pour lesquelles les individus se tatouent sont nombreuses, variées, souvent intriquées et surtout personnelles. Le tatouage pour camoufler des cicatrices ou des lésions cutanées dyschromiques et disgracieuses est pratiqué de longue date [2,3]. Ainsi, le tatouage médical [4], aussi dénommé « micropigmentation », « dermopigmentation » ou « dermatographie », a été utilisé pour camoufler des cicatrices post-traumatiques, des cicatrices de brûlures, des greffes de peau [5] et certaines dermatoses comme une alopécie, une dépigmentation (vitiligo, piebaldisme), voire dans le passé les angiomes plans.

Le tatouage occupe une place importante en sénologie, lors de la reconstruction de la plaque aréolo-mammaire (PAM) après mastectomie [4,6]. Elle fait partie intégrante de la prise en charge de la patiente. Elle annonce la fin du processus de reconstruction qui a souvent été long et parfois difficile à vivre pour la patiente et va améliorer de façon significative la perception de son image corporelle. Le bénéfice de la reconstruction de l'aréole mammaire est reconnue [6]. La reconstruction de la PAM n'est pas restreinte au cancer. Les anomalies congénitales comme l'athélie ou l'amastie, les traumatismes ou les brûlures sont autant d'indications pour un tatouage.

Les médecins, les chirurgiens [7] ou parfois leurs infirmières, les esthéticiennes mais aussi les tatoueurs pratiquent ces tatouages de camouflage ou de reconstruction (Fig. 1). Au final, la seule différence séparant le corps médical des tatoueurs est que ces derniers peuvent laisser libre court à leur imagination et réaliser des tatouages artistiques colorés [8].

Depuis quelques années, une «mode » quelque peu différente est apparue. Des patientes n'hésitent plus à se parer de tatouages véritablement décoratifs en lieu et place d'un « simple » tatouage de la PAM (Fig. 2). La fréquence de cette pratique n'est pas connue. Cependant, une simple recherche sur Google en utilisant les termes mastectomy tattoos donnent une idée de l'importance de cette pratique. Les réseaux sociaux, facebook, instagram ou surtout pinterest, un réseau social visité majoritairement par un public féminin [9] ainsi que les sites grand public sur Internet [10] contribuent à les rendre encore plus populaires. Un site www.p-ink.org est même dédié à mettre en relation des patientes avec des tatoueurs. Ainsi s'affichent des femmes fières avec leur(s) poitrine(s) décorées de motifs floraux ou animaliers (bien souvent des oiseaux). Plus rarement, il s'agit de tatouages couvrant ou épousant essentiellement une cicatrice.

\section{Les bénéfices}

Les bénéfices sont multiples. Par sa fonction première, le tatouage permet de camoufler les cicatrices opératoires et l'absence de PAM. Tatouer sur une cicatrice cachera la

\footnotetext{
Il s'agit d'un éditorial sur une mode de plus en plus fréquente, les tatouages décoratifs sur mastectomies après cancer du sein.

$\diamond$ This is an editorial about a recent trend and its benefits, decorative tattooing on mastectomy for breast cancer.
} 


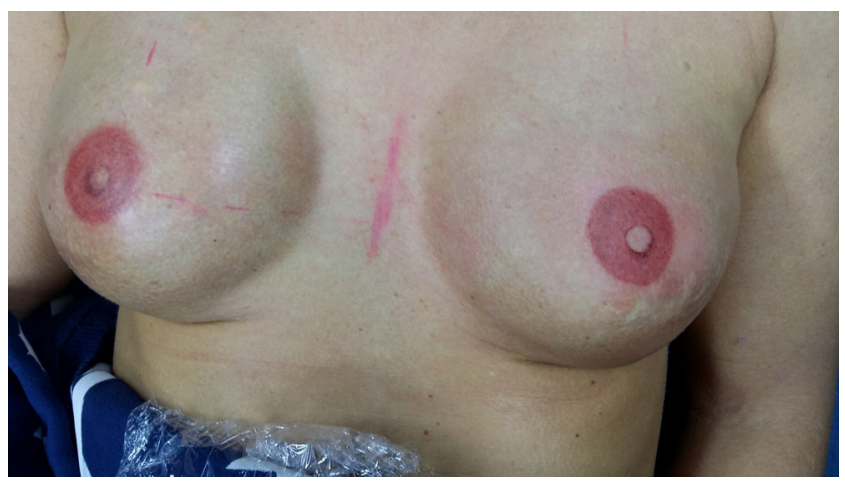

Figure 1 Tatouage des 2 plaques aréolo-mammaire de reconstruction chez une patiente ayant subie une double mastectomie préventive. Notez le résultat du téton gauche. Collection Manao Tiki Tattoo, Ollioules.

dyschromie, mais ne permet pas d'aplanir cette dernière. Il n'est pas toujours nécessaire de tatouer dessus. On peut ainsi intégrer une cicatrice de façon astucieuse au tatouage afin d'attirer l'œil de l'autre sur ce dernier et non plus sur la cicatrice. De même, il permet de cacher l'absence de sein en cas de mastectomie totale. Cependant, le tatouage est ici appliqué sur une zone cachée, qui n'est visible que de la patiente elle-même, devant un miroir, ou dans l'intimité du couple. Comme tout tatouage, ce dernier a une fonction naturellement esthétique [11] et d'embellissement du corps, d'un corps ici malade et mutilé par une chirurgie nécessaire. Mais, il est reconnu que le tatouage permet de
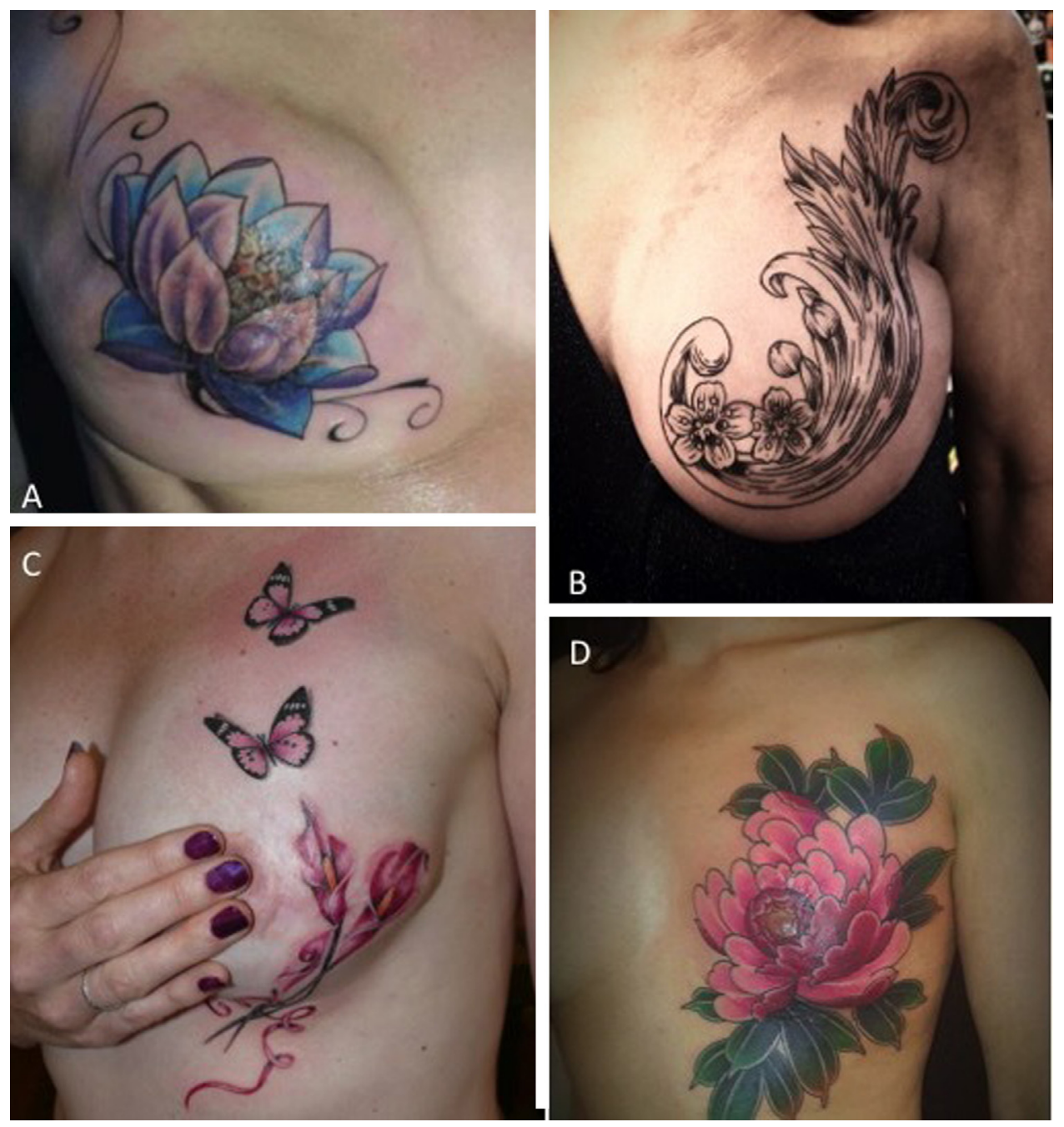

Figure 2 Exemples de tatouages décoratifs après cancer du sein par des tatoueurs professionnels. A. L'entre peau, Argences. B. Viscéral, Aix-en-Provence. C. Gaelle, Dermoglam, Lannion. D. Lucien Monsieur Biz, Aix-en-Provence. 
renforcer l'ego et de compenser les insuffisances perçues par les individus [12], insuffisances qui semblent assez évidentes dans le cas de patientes avec une mastectomie. Le tatouage permet indéniablement à ces dernières de reprendre le contrôle de leur corps, de maîtriser cette partie du corps qui leur échappe. Le tatouage apporte une amélioration de l'image corporelle chez les patients et une augmentation de la confiance en soi (ego bolstering) [12]. Cette reprise du contrôle du corps via les tatouages n'est pas l'apanage des tatouages sur mastectomie. Elle est observée chez de nombreux patients. Dans notre expérience, nombreux patients usent de cette stratégie d'adaptation (ou coping). Ainsi, de nombreux patients avec un diabète de type I se font tatouer leur maladie sous la forme de tatouages décoratifs mentionnant leur maladie, en lieu et place du traditionnel bracelet d'alerte mais aussi pour reprendre un contrôle sur leur maladie [13]. Il peut également s'agir de maladies génétiques diverses [14] ou de dermatoses chroniques comme un psoriasis ou un vitiligo. Le tatouage rapproche les individus : il permet également de sensibiliser les autres, les amis, les relations de travail ou même les anonymes dans la rue [13-15]. De façon intéressante, les proches, qui sont opposés aux tatouages et expriment souvent une réticence initiale, acceptent bien souvent le tatouage a posteriori avec un fort soutien [13], comme si le tatouage devenait acceptable pour cette raison. Le choix du motif et du type du tatouage est variable selon l'histoire de chacun, mais sur les réseaux sociaux, les fleurs, qui expriment la féminité, ou les oiseaux, pour l'envol ou la renaissance, sont légions. De plus, de fait de la forme ronde des seins, des volutes sont souvent utilisés car un tatouage se doit de respecter une certaine dynamique qui épouse l'anatomie de la femme (Fig. 2).

\section{Les complications et les risques}

\section{Les complications non-spécifiques}

Elles sont propres à n'importe quel tatouage indépendamment du terrain de la patiente. Elles ont été développées en détail dans de nombreux articles $[16,17]$.

Avant tout, les risques sont ceux d'un tatouage disgracieux, qui ne correspond pas aux souhaits de la patiente, et le regret. Elles imposent que le tatouage soit planifié et mûrement réfléchi, ce qui est toujours le cas chez ces patientes, car le tatouage ne peut être réalisé qu'en fin de traitement, chez une patiente en rémission, à distance de la chimiothérapie. De plus, il convient d'attendre au moins une année après la radiothérapie avant de se faire tatouer. Le tatouage doit être réalisé chez un professionnel dans un studio. Ce dernier doit être prévenu tout comme l'oncologue qui suit la patiente du désir du tatouage.

Les risques médicaux associés au tatouage sont les mêmes que pour tout un chacun : infection cutanée, réaction allergique à une couleur, localisation d'une dermatose chronique $[16,17]$.

\section{Les risques spécifiques}

Les risques évoqués ici sont à ce jour théorique, car aucun cas n'a été rapporté du fait de la nouveauté de cette pratique. Il s'agit donc ici d'explorer et discuter les possibles risques sur un tel terrain particulier.

La question des risques carcinogènes in vivo des encres est encore ouverte. Une résolution du Conseil de l'Europe émise en 2008 (et transcrit dans le droit français) définit une liste de composants interdits dans les encres européennes [18]. Un rapport très complet vient d'être publié fin juillet 2016 par la commission européenne [19] et cette dernière a mandaté l'agence chimique européenne (ECHA) pour évaluer les produits devant être exclus des encres. Cependant, il faut rappeler qu'à ce jour, il n'existe pas de lien entre cancer de peau et tatouage permanent [20]. Des cas ont certes été rapportés, mais il s'agit plus d'un bruit de fond à mettre en relation avec l'augmentation observée des cancers de peau, dont les mélanomes. Des cas de kératoacanthomes uniques ou multiples ont été décrits essentiellement en regard du rouge des tatouage [20].

Les risques de production de composés de dégradation pro-carcinogène dans les suites d'une deuxième session de radiothérapie à travers la peau tatouée est à jour hypothétique. Elle ne peut et ne doit bien sûr pas contre-indiquer la radiothérapie, si cette dernière est indispensable. De plus, il semble quelque peu inconvenant de se poser la question d'un hypothétique risque de cancer secondaire chez une patiente elle-même en récidive d'un cancer !

La question du passage systémique de composants des encres ou de produits de décomposition est là aussi en suspens. De plus, les patientes ont subi un curage ganglionnaire axillaire. Les macrophages présents dans la peau tatouée migrant dans les vaisseaux lymphatiques ne sont pas arrêtés dans les ganglions régionaux. Le risque de passage systémique de composés dans la circulation et in fine dans certains organes est inconnu.

Il est important de rappeler que les tatoueurs professionnels qui réalisent des tatouages de PAM (Fig. 1) utilisent les mêmes encres/marques que celles qu'ils utilisent pour leurs tatouages décoratifs, si bien qu'au final, les questionnements sont les mêmes pour les deux types de tatouages.

Au total, la pratique du tatouage décoratif sur mastectomies apparaît comme une solution pour certaines femmes. Bien qu'il existe quelques zones d'obscurité sur les risques carcinogènes des encres in vivo, ces risques nous semblent à ce jour grandement théoriques et surtout minimes comparés aux bénéfices en termes de bien-être psychologique. Les motivations qui les poussent vers ce choix spécifique plus qu'un tatouage « classique » de reconstruction de PAM n'ont pas fait l'objet d'études. Elles sont probablement en relation avec un contact avec le tatouage dans le passé ou dans leur environnement (ami(es) tatoué(es), etc.). Toutes ne souhaitent certainement pas se tatouer et il ne s'agit ici pas de pousser toutes les patientes vers cette solution ! En revanche, le bénéfice psychologique d'un tel résultat est indéniable comme dans d'autres maladies. Que l'on soit « pour» ou « contre » les tatouages, il faut savoir écouter ces patientes et les conseiller le mieux possible pour leur permettre d'aller jusqu'au bout.

\section{Déclaration de liens d'intérêts}

Nicolas Kluger est membre d'honneur du Syndicat national des artistes tatoueurs (SNAT). 


\section{Remerciements}

L'auteur remercie les tatoueurs membres du Syndicat national des artistes tatoueurs pour leur disponibilité à fournir de nombreux clichés de tatouages sur mastectomies, dont seulement certains ont été choisis ici.

\section{Références}

[1] Fourquet J. Les Français et les tatouages. Sondage Ifop; 2010, http: / /www.ifop.com/?option=com_publication\&type= polleid $=1220$.

[2] Bruno C. Tatoués, qui êtes-vous?. Éditions De Feynerolles; 1974

[3] Grognard C, Froge E. Le tatouage : illustration, réparation. Paris: Ed. Arnette; 1991.

[4] Vassileva S, Hristakieva E. Medical applications of tattooing. Clin Dermatol 2007;25:367-74.

[5] Byars LT. Tattooing of free skin grafts and pedicle flaps. Ann Surg 1945;121:644-8.

[6] Riot S, Devinck F, Aljudaibi N, Duquennoy-Martinot V, Guerreschi $P$. Tatouage de la plaque aréolo-mammaire en reconstruction mammaire : note technique. Ann Chir Plast Esthet 2016; 61:141-4.

[7] Kim EK, Chang TJ, Hong JP, Koh KS. Use of tattooing to camouflage various scars. Aesthetic Plast Surg 2011;35:392-5.

[8] Spyropoulou GA, Fatah F. Decorative tattooing for scar camouflage: patient innovation. J Plast Reconstr Aesthet Surg 2009;62:e353-5.

[9] Dugan M. The demographics of social media users; 2015, http://www. pewinternet.org/2015/08/19/ the-demographics-of-social-media-users/.

[10] Kylstra C. 13 powerful photos of mastectomy tattoos; 2014, https://www.buzzfeed.com/carolynkylstra/mastectomytattoos?utm_term=.ueb7LL3G9\#.wbZGee2xq.

[11] Wohlrab S, Stahl J, Kappeler PM. Modifying the body: motivations for getting tattooed and pierced. Body Image 2007;4:87-95.
[12] Raspa RF, Cusack J. Psychiatric implications of tattoos. Am Fam Physician 1990;41:1481-6.

[13] Kluger N, Aldasouqi S. The motivations and benefits of medical alert tattoos in patients with diabetes. Endocr Pract 2013; 19:373-6.

[14] Whittaker RG, Turnbull DM. A diagnostic tattoo. Clin Genet 2009;75:37-8.

[15] Kluger N, Aldasouqi S. A new purpose for tattoos: medical alert tattoos. Presse Med 2013;42:134-7.

[16] Kluger N. Cutaneous complications related to permanent decorative tattooing. Expert Rev Clin Immunol 2010;6:363-71.

[17] Boulart L, Mimoun M, Noel W, Malca N, Chaouat M, Boccara D. Autour du tatouage en chirurgie plastique. Complications et tatouage médical. Ann Chir Plast Esthet 2016 [sous presse].

[18] Rico AM. EU actions to ensure the safety of tattoos and the protection of consumers. Curr Probl Dermatol 2015;48:206-9.

[19] Piccinini P, Pakalin S, Contor L, Bianchi I, Senaldi C. Safety of tattoos and permanent make-up. Final report. Administrative Arrangement No. 2014-33617 Analysis conducted on behalf of DG JUST. https://ec.europa.eu/jrc/en/publication/eurscientific-and-technical-research-reports/safety-tattoosand-permanent-make-final-report.

[20] Kluger N, Koljonen V. Tattoos, inks, and cancer. Lancet Oncol 2012;13(4):e161-8.

N. Kluger

Departments of dermatology, allergology and venereology, university of Helsinki and Helsinki university Central Hospital, 00029 Hus, Finlande

Adresse e-mail : nicolas.kluger@hus.fi

Reçu le 17 août 2016 Accepté le 14 septembre 2016 